\title{
Power Aware Connection Provisioning For All-Optical Multicast Traffic in WDM Networks
}

\author{
Ashraf M. Hamad and Ahmed E. Kamal
}

\begin{abstract}
The connection provisioning problem attempts to achieve its objective of guaranteeing the maximum throughput and minimal blocking probability. In optical networks, this problem is mainly the classical Routing and Wavelength Assignment (RWA) problem, which includes many constraints. In this study, we investigate the RWA problem for connection provisioning under multicast traffic while considering the optical power constraints. The problem is first formulated as a MixedInteger Linear Program (MILP) with the objective of minimizing the session blocking rate. In order to provide fast and efficient solutions, the paper introduces a novel heuristic solution which divides the problem into subproblems and solves them separately, while still taking the interdependency between them into account. The results obtained from both solutions are found to be closely comparable. The results obtained from the heuristic also provide an insight for the network operators about the maximum performance enhancement that can be achieved by upgrading the network capacity.
\end{abstract}

Index Terms-All-Optical Multicasting, Power Aware Multicasting, Routing and Wavelength Assignment (RWA).

\section{INTRODUCTION}

W AVELENGTH routing WDM optical networks providing all-optical service has become the most viable technology for future wide-area backbone networks. On one hand, the huge bandwidth of these networks is the answer for the exponential growth in traffic demands that is due to the increase in the number of users and bandwidth extensive applications. On the other hand, the all-optical scheme supports the various traffic types and services optically by eliminating any conversion of the transport signal between the electronic and optical domains at intermediate nodes. Such elimination for the signal conversion has the following advantages:

1) Reducing nodes costs and complexity by removing the need to use signal convertors at intermediate nodes.

2) Achieving signal transparency by removing the need to know the signal type, bit rates and protocol used to transmit the signal, and

3) Simplifying the logical network stack structure.

The Routing and Wavelength Assignment (RWA) problem [1] is one of the fundamental problems in wavelength routed networks. The general version of this problem is NP-hard as it encompasses finding feasible routes and wavelength(s) assignment while optimizing one or more performance measures. Depending on the traffic type, the session route can be a path, a tree or a forest. On the other hand, the performance

Ashraf Hamad is with Microsoft Corporation, One Microsoft Way, Redmond, WA 98052-6399, USA; Email: ahamad@microsoft.com.

Ahmed Kamal is with Department of Electrical and Computer Engineering; Iowa State University, Ames, IA 50011-3060, USA; Email: kamal@iastate.edu. measures in the objective function depend on the problem context. In one context, namely, Network Provisioning, the problem is a network design problem with the objective of minimizing the network cost. In another context, namely, Connection Provisioning, the problem is studied as a network operation problem and its goal is to maximize the network throughput, minimize the wavelength usage, or minimize the session blocking probability.

In some cases, the RWA is formulated to include more realistic constraints, which makes it more complicated. One of these constraints is power impairments, which include the: Optical Amplifiers (OAs) gain model, power loss and noise. This formulation adds a new dimension to the classical RWA problem, namely, the power dimension. We refer to this problem as Power-Aware RWA (PA-RWA).

Since optical signals suffer from power loss due to propagation in optical fibers, PA-RWA is important for all traffic types. However, it is more critical for All-Optical Multicasting (AOM) Service [2][3] as the optical signal in this case undergoes additional power loss. The source of this extra power loss is the splitting of the power strength of the incoming signal at the branching nodes of the multicast delivery structures (lighttrees [4] or light-forests [5]) into multiple output links. Power splitting is done in the optical domain using configurable passive optical splitters.

In this paper, we investigate the Connection Provisioning aspect of the PA-RWA problem for AOM traffic. We first formulate the PA-RWA problem as a Mixed Integer Linear Program (MILP) with the objective of minimizing the total number of blocked sessions. Like [6], our MILP formulation handles the case of the unequally powered signals at the links/OAs entries. However, the formulation in [6] is a Mixed Integer Non-Linear Program (MInLP) that deals with unicast traffic only in optical LAN/MAN networks. Our formulation here has the advantage of being a linear solution for the more general mesh network topology and multicast traffic. Nevertheless, the MILP is neither fast nor scalable for large problem instances. Therefore, a greedy heuristic that provides fast and near optimal solutions for the PA-RWA problem is then introduced.

The remainder of the paper is organized as follows. Next section overviews the literature of the PA-RWA problem. Section III defines the PA-RWA problem in the Connection Provisioning context and introduces the system model. This is followed by the MILP formulation and heuristic details in Sections IV and V, respectively. Numerical results are presented in Section VI and the paper is concluded in Section VII. 


\section{RELATED WORK}

The PA-RWA problem has been studied in the literature in the Network Provisioning and Connection Provisioning contexts. In the former context, the primary cost factor of the backbone network is the cost of the OAs. Hence, PA-RWA is strongly coupled with the Optical Amplifier Placement (OAP) problem which finds and places the minimum number of OAs in the network. As such, the coupled problem consists of three integrated subproblems, namely, the Routing (R), Wavelength Assignment (WA) and Power Assignment (PA) subproblems.

To address these subproblems, the authors introduced a Mixed Integer Linear Program (MILP) in [7] that solves the $\mathrm{R}$, WA and PA subproblems in an integrated fashion for AOM traffic. The network cost in [7] is represented as the total power amplification needed in the network. The MILP formulation was extended in [8] to determine the exact number of OAs instead of the total amplification. We also proposed a heuristic approach in [8] and [9], called Optical amplifier Placement (OP) algorithm, to provide fast and efficient solutions by employing two schemes. The first scheme uses alternate routing for light-forest construction while the second scheme allows light-forest reconstruction. Results show that using the adaptive scheme alone outperforms the alternate routing scheme, and utilizes fewer network resources.

For unicast traffic, reference [10] addressed the PA-RWA problem in broadcast-and-select networks where no routing is performed. The problem was formulated as a MInLP with the objective of minimizing the total number of OAs while considering the case of unequally powered-signals at the entry points of the OAs. The same problem was then solved in [11] using MILP while relaxing this condition and assuming that the signals are equally powered at the OA entry points. The work in [12] generalized the problem in [10] by incorporating different layout topologies (stars, trees and/or rings) and by taking into account the fact that the cost of OAs is location dependent. The problem was solved using simulated annealing, which was also used by the authors in [13] in order to jointly solve the RWA and OAs placement problems in WDM networks that are based on existing power grids that are used to distribute electricity.

The second aspect of the PA-RWA problem, namely, Connection Provisioning, studies the impact of power constraints during the operation phase of already provisioned networks. In [14], an iterative heuristic was proposed in which an initial tree is constructed without taking any power constraint into consideration. This tree is then modified by replacing a set of adjacent splitters by a single splitting node, called the Centralized Splitting Node, in order to reduce the total amount of power loss. Another heuristic algorithm is proposed in [15] which ensures a minimum signal quality and fairness among all destinations by using the concept of balanced light-trees.

The problem was also studied in the context of unicast traffic. The authors in [6] proposed a MInLP for PA-RWA in order to minimize the sessions blocking probability. A two-phase hybrid solution employing a greedy heuristic and Genetic Algorithm for session establishment and power assignment was introduced.
On the other hand, the work in [16] proposed a heuristic approach for PA-RWA that takes into account a number of linear and non-linear power impairments, including ASE noise, crosstalk, filtering effects, cross phase modulation and four wave mixing. The algorithm is based on assigning a cost function for the links that integrates all different types of impairments in the signal quality. These cost factors are then used to compute the RWA solutions of the sessions.

\section{PROBLEM DESCRIPTION}

In this section, we first present the power constraints and the OA gain model we use in our solutions. We then introduce the system model and assumptions in Subsection III-B. Finally, we formally define the PA-RWA problem in Subsection III-C.

\section{A. Power Constraints and Optical Amplifier Gain Model}

Our solutions handle two main system power constraints:

1) The power strength of each wavelength must not fall below a certain threshold, called $P_{S e n}$ at any point in the network. This constraint is needed to ensure that these signals are detectable by the receivers and OAs.

2) The aggregate power values of all wavelengths on a fiber must not exceed an upper bound, called $P_{M A X}$, in order to maintain the system within the linear operation region of the fibers.

We also use a simple, but accurate, piece-wise model for the OA gain which is expressed as:

$$
G\left(P_{i n}\right)=\operatorname{MIN}\left\{G_{0},\left(P_{M A X}-P_{i n}\right)\right\}
$$

where $P_{i n}$ represents the aggregate power of the input signal, and $G_{0}$ is the small-signal gain in $d B$. Both $P_{M A X}$ and $P_{i n}$ are in $d B m$. Assuming flat gain over all the channels, this gain applies to all input wavelengths.

\section{B. System Model and Assumptions}

The network is an all-optical wavelength-routed WDM mesh network and is represented as a undirected connected graph. Each undirected edge in the graph is equivalent to two fibers (links) carrying traffic in opposite directions and all fibers support the same set of wavelengths. Each vertex represents an optical cross connect (OXC) with Drop-andContinue (DaC) capability [2], where portion of the power strength (denoted by $\gamma$ ) of the passing signal is tapped and terminated at the node. The deployment of the splitters at the nodes is sparse. Nodes that are equipped with power splitters are called Multicast capable (MC) nodes; otherwise, the nodes are called Multicast Incapable (MI) nodes. The splitters have complete (i.e., splitting fanout of the node is equal to its outdegree) and fixed (i.e., the input signal will be split equally among the output ports) splitting capabilities.

Also, the network does not support wavelength conversion; hence, wavelength continuity constraints should be enforced. Moreover, the network is pre-provisioned; therefore, the number and placement of the OAs is pre-determined and known. Also, each node is equipped with an array of a sufficient number of fixed-tuned transceivers (transmitters/receivers). 
Using the notation introduced in [2], our system model is characterized as $S^{s} F^{c} R^{x}-C^{n}$. This notation consists of two parts, each of which describes one aspect of the network model. The terms in the first part describe the splitting capability of the nodes, such that:

- $S^{s}$ indicates sparse $(s)$ splitting $(S)$ deployment,

- $F^{c}$ indicates complete $(c)$ fan-out $(F)$ of the splitters, and

- $R^{x}$ specifies that the splitting ratio $(R)$ is fixed $(x)$.

The second term indicates that the wavelength conversion capability $(C)$ is absent (hence, the superscript $n$ is used).

The network traffic demands are static and of the multicast type. As a result of the sparse splitting and the absence of wavelength conversion capability, the multicast delivery structure can take the form of light-forests. In our study, each light-tree of the light-forest is rooted at the source node and launched using a single dedicated transmitter. A multicast session is considered accepted if and only if all nodes in its destination set are reachable by the light-forest (i.e., full destination delivery acceptance policy).

For the sake of simplicity, our study takes into account three sources of power loss, namely, propagation, splitting and tapping losses. Other loss sources and impairments due to nonlinearities and noise are outside the scope of this work. We assume all power levels are in $d B m$, while power gain/loss are in $d B$. Finally, the value of $P_{\text {sen }}$ is assumed to be high enough to cope with the various types of noise and to guarantee an adequate Bit Error Rate (BER) [17].

\section{Problem Definition}

Definition: Given the network topology, static multicast traffic demands, number and locations of the splitters and OAs, number of wavelengths, the Power-Aware Routing and Wavelength Assignment (PA-RWA) problem under AOM is defined as a connection provisioning problem whose objective is to find a feasible light-forest (i.e., route) and wavelength(s) assignment for the maximum number of multicast sessions in order to minimize the connection blocking rate.

\section{MILP PROBlem Formulation}

We introduce the network parameters and formulation variables in Subsections IV-A and IV-B, respectively. The objective function and constraints are presented in Subsection IV-C.

\section{A. Network Parameters}

The following network input parameters are used in the MILP formulation:
$N, E \quad$ The set of nodes, and fiber links, respectively.
$\Lambda \quad$ The set of wavelengths supported per fiber.
$i, j, k \quad$ Node identity, where $i, j, k \in N$.
$\lambda \quad$ Wavelength identity, where $\lambda \in \Lambda$.
$e(i, j) \quad$ Fiber link directed from node $i$ to node $j$.
$O A_{i, j} \quad$ The set of optical amplifiers on fiber $e(i, j)$.
$N O A_{i, j} \quad$ Binary indicator: 1 if $\left|O A_{i, j}\right|>0 ; 0$ otherwise.
$o \quad$ OA identity on $e(i, j) ; 1 \leq o \leq\left|O A_{i, j}\right|$.
$S P \quad$ The set of splitters.
$S P_{i} \quad$ Binary indicator: 1 if node $i$ has a splitter.
$\beta \quad$ Propagation loss ratio $(d B / \mathrm{km})$ over the fiber.

$\gamma \quad$ Tapping power loss value $(d B)$ at each node, which is due to the node's $\mathrm{DaC}$ capability.

$L_{i, j} \quad$ Length $(\mathrm{km})$ of fiber $e(i, j)$.

$L_{i, j}^{x, y} \quad$ Length $(\mathrm{km})$ between sites $x$ and $y$ of $e(i, j)$. $x=0\left(y=\left|O A_{i, j}\right|+1\right)$ refers to node $i(j)$.

$G_{0} \quad$ Small signal gain achieved for non-saturated OA.

$K \quad$ Number of multicast sessions.

$a \quad$ Multicast session identity; $0 \leq a \leq K-1$.

$\operatorname{src}_{a} \quad$ Source node of multicast session a.

$D_{a} \quad$ Destination set of multicast session a.

$\Phi_{i} \quad$ Set of sessions in which node $i$ is a destination.

$\Gamma_{i}^{a} \quad$ Binary indicator: 1 if $i \in D_{a}$; 0 otherwise.

$P_{S e n}^{d B m} \quad$ Power sensitivity strength $(\mathrm{dBm})$ on a channel.

$P_{S W W}^{m W}$

$P_{S e n}$

MAX

$T P_{M A X}^{m W}$
$T P_{M W}^{m W}$

$T P_{M I N}^{m W}$

$P_{1}, P_{2}$

Power sensitivity strength $(\mathrm{mW})$ on a channel.

Maximum power $(\mathrm{dBm})$ for each channel.

Maximum aggregate power ( $\mathrm{mW}$ ) on any link.

Minimum aggregate power $(\mathrm{mW})$ on any link.

Two negative constants that are used to represent the case of $(-\infty) d B m$, such that $P_{1}<P_{2}$.

$\delta \quad$ Very small number used for mapping purposes.

$v, w \quad$ Integer constants used for calculating the power values.

Out ${ }_{i} \quad$ Out degree of node $i$.

$M \quad$ Very large number that is used as a standard approach in MILP to enforce conditional rules on the constraints; where $M>\operatorname{MAX}\left\{N^{2} *|\Lambda|, G_{0}\right\}$.

\section{B. MILP Variables}

The following variables are used in the MILP formulation:

$W_{1}, W_{2} \quad$ Weights of objective function factors; $W_{1} \gg W_{2}$.

$T_{i, j}^{a, \lambda} \quad$ Binary indicator: 1 if $e(i, j)$ is used by session $a$ over $\lambda$; 0 otherwise.

$\varphi_{a} \quad$ Binary indicator: 1 if session $a$ is provisioned in the network; 0 otherwise.

$\vartheta_{i}^{a} \quad$ Binary indicator: 1 if node $i$ is included in the light-forest of session $a$; 0 otherwise.

$\Im_{i, j}^{a} \quad$ Binary indicator: 1 if $e(i, j)$ is used by session $a$ over any wavelength; 0 otherwise.

$\Upsilon_{i, j} \quad$ Binary indicator: 1 if $e(i, j)$ is used by any session over any wavelength; 0 otherwise.

$G_{i, j}^{o} \quad$ Value of the amplification gain required from the $o^{\text {th }}$ optical amplifier on fiber $e(i, j)$.

$S L_{i}^{a, \lambda} \quad$ Splitting loss on channel $\lambda$ at node $i$ for $a$.

$\Omega \quad$ Power inspection site; $\Omega \in\{$ beg, end $\}$.

$P_{i, j, d B m}^{\Omega, a, \lambda}$

$P_{i, j, m W}^{b e g, a, \lambda, o} \quad$ Power level (mW) at beginning of $o^{\text {th }}$ OA on

Power level $(\mathrm{dBm})$ at beginning $(\Omega=b e g) /$ end $(\Omega=e n d)$ of $e(i, j)$ for $\lambda$ used by session $a$.

$T P_{i}^{\text {beg }} \quad$ Total power (mW) at beginning of $e(i, j)$.

$T P_{i, j, m W}^{b e g, o}$ Total power (mW) at entry of $o^{t h} \mathrm{OA}$ on $e(i, j)$.

$f$

$A_{f}$

$A P_{i, j}^{o} \quad$ Binary indicator used for OA gain linearization. $Y$
Tree fanout; number of outgoing tree links.

Binary indicator used for power splitting linearization.

Set of linear equations that are selected for unit conversion between $m W$ and $d B m / d B$. 


\section{MILP Formulation}

The objective function is to minimize the number of blocked sessions in the network, and is expressed as follows:

$$
\text { Minimize: } \quad-W_{1} \sum_{a=0}^{K-1} \varphi_{a}+W_{2} R
$$

where $R$ is expressed as follows:

$$
\begin{aligned}
R=\sum_{e(i, j) \in E} \sum_{o \in O A_{i, j}} \sum_{a=0}^{K-1} \sum_{\lambda \in \Lambda} T P_{i, j, m W}^{b e g, a, \lambda, o}+ & \sum_{e(i, j) \in E} \sum_{o \in O A_{i, j}} G_{i, j}^{o}
\end{aligned}
$$

The rationale behind using $R$ in the objective function is to simplify the evaluation of the value of the total power per link and the OA gain variables, as will be shown below. The weight $W_{2}$ is chosen such that its value is much less than $W_{1}$. This is essential to ensure that the contribution of $R$ to the value of the objective function is much less than that of the number of admitted sessions.

The objective function is subject to a set of constraints, which are discussed below:

\section{Routing and Wavelength Assignment Constraints}

The construction of the light-forest and its wavelength(s) assignment are determined by the following constraints:

$$
\begin{gathered}
\varphi_{a} \leq \sum_{i, i \in D_{a}} \frac{\vartheta_{i}^{a}}{\left|D_{a}\right|} \quad 0 \leq a<K \\
\varphi_{a} \geq \sum_{i, i \in D_{a}} \vartheta_{i}^{a}-\left(\left|D_{a}\right|-1\right) \quad 0 \leq a<K
\end{gathered}
$$

The purpose of these constraints is to enforce the full destination delivery policy by ensuring that session $a$ is accepted if all its destinations are part of the session's light-forest. Constraint (4) guarantees that $\varphi_{a}=0$ if at least one destination node is not part of the light tree (namely, if the right-hand side of the equality is less than 1). However, constraint (4) does not guarantee that $\varphi_{a}=1$ if its right-hand side equals 1. Therefore, constraint (5) is needed to ensure that $\varphi_{a}=1$ when the right-hand side of constraint (5) is 1 . These two constraints together compute $\varphi_{a}$ as the conjunction between all $\vartheta_{i}^{a}$ variables of session $a$ 's destination nodes.

$$
\begin{aligned}
& \vartheta_{j}^{a} \geq \sum_{i, i \neq j, e(i, j) \in E} \sum_{\lambda \in \Lambda} \frac{T_{i, j}^{a, \lambda}}{M} \forall j \in N ; 0 \leq a<K \\
& \vartheta_{j}^{a} \leq \sum_{i, i \neq j, e(i, j) \in E} \sum_{\lambda \in \Lambda} T_{i, j}^{a, \lambda} \forall j \in N ; 0 \leq a<K
\end{aligned}
$$

Constraints (6) and (7) set $\vartheta_{j}^{a}$ to the disjunction between all $T_{i, j}^{a, \lambda}$ variables, for all incoming links from the neighboring nodes of node $j$ over all wavelengths. It indicates that node $j$ is part of sessions $a$ 's light-forest if it is part of at least one of its light-trees.

$$
\Im_{i, j}^{a} \geq \sum_{\lambda \in \Lambda} \frac{T_{i, j}^{a, \lambda}}{M} \quad \forall i, j \in N ; 0 \leq a<K
$$

$$
\Im_{i, j}^{a} \leq \sum_{\lambda \in \Lambda} T_{i, j}^{a, \lambda} \quad \forall i, j \in N ; 0 \leq a<K
$$

Constraints (8) and (9) are used to determine if link $e(i, j)$ is used by session $a$. $\Im_{i, j}^{a}$ is set to 1 if a tree used by session $a$ traverses link $e(i, j)$ over any wavelength. $\Im_{i, j}^{a,}$ is evaluated as the disjunction between the $T_{i, j}^{a, \lambda}$ variables for session $a$.

$$
\Upsilon_{i, j} \geq \sum_{a=0}^{K-1} \frac{\Im_{i, j}^{a}}{M} ; \Upsilon_{i, j} \leq \sum_{a=0}^{K-1} \Im_{i, j}^{a} \quad \forall e(i, j) \in E
$$

Constraint (10) sets $\Upsilon_{i, j}$ to the disjunction between all $\Im_{i, j}^{a}$ variables, for all sessions. It indicates that link $e(i, j)$ is used by at least one session.

$$
\begin{array}{r}
\frac{\Gamma_{i}^{a}+\sum_{\lambda \in \Lambda} \sum_{e(i, k) \in E} T_{i, k}^{a, \lambda}}{M} \leq \sum_{\substack{e(k, i) \in E \\
\forall i, i}} T_{k, i}^{a, \lambda}+\left(1-\varphi_{a}\right) \times M \\
\forall i \in N /\left\{\operatorname{src}_{a}\right\} ; 0 \leq a<K(11)
\end{array}
$$

The above constraint is used to create the light-forest per session by ensuring the reachability of each of its destinations. This is achieved by ensuring that there should be at least one incoming link to node $i$ if it is a destination or it leads to a destination node, only if session $a$ is accepted, i.e., $\varphi_{a}=1$.

$$
\sum_{a=0}^{K-1} \sum_{e(i, j) \in E} T_{i, j}^{a, \lambda} \leq 1 \quad \forall i, j \in N ; i \neq j ; \forall \lambda \in \Lambda
$$

This constraint guarantees that wavelength $\lambda$ is used on link $e(i, j)$ by at most one light-tree.

$$
\begin{aligned}
& \sum_{e(i, j) \in E} T_{i, j}^{a, \lambda} \leq 1 \\
& \quad \forall i \in N /\left\{s r c_{a}\right\} ; i \notin S P ; 0 \leq a<K ; \forall \lambda \in \Lambda
\end{aligned}
$$

Constraint (13) prevents branching at MI nodes by ensuring that any MI node, say $i$, has at most one outgoing tree link over a particular wavelength.

$$
\begin{aligned}
& \sum_{e(i, j) \in E} T_{i, j}^{a, \lambda} \geq \sum_{\substack{e(j, k) \in E \\
\forall j \in N /\left\{s r c_{a}\right\}}} \frac{T_{j, k}^{a, \lambda}}{M} \\
& \quad \forall j a<K
\end{aligned}
$$

Constraint (14) is used to guarantee wavelength continuity for each session. This is achieved by ensuring node $j$ should have an incoming tree link over wavelength $\lambda$ if node $j$ has at least one outgoing link employing the same wavelength.

\section{Loop-Avoidance Constraints}

The number of fiber hops used to reach a node from the source of the light-forest is used to prevent loops from developing. In this paper, we use the same set of constraints we developed for the OAP problem in [8]. Due to space limitation, the constraints are not included here, and the reader is referred to constraints (14)-(16) in [8].

\section{Power Constraints}

The following are the power constraints:

$$
\begin{aligned}
& P_{i, j, d B m}^{b e g, a, \lambda, o} \geq P_{S e n}^{d B m} \times T_{i, j}^{a, \lambda}+P_{1} \times\left(1-T_{i, j}^{a, \lambda}\right) \\
& \forall e(i, j) \in E ; 0 \leq a<K ; \forall \lambda \in \Lambda ; \forall o \in O A_{i, j}
\end{aligned}
$$




$$
\begin{array}{r}
P_{i, j, d B m}^{e n d, a, \lambda} \geq P_{S e n}^{d B m} \times T_{i, j}^{a, \lambda}+P_{1} \times\left(1-T_{i, j}^{a, \lambda}\right) \\
\forall e(i, j) \in E ; 0 \leq a<K ; \forall \lambda \in \Lambda
\end{array}
$$

These constraints ensure that the power levels at the entrance of an OA, i.e, equation (15), and the end of a fiber, i.e., equation (16), are above $P_{S e n}^{d B m}$ if the link is used by the lightforest. Enforcing this condition at these points is sufficient to ensure that this lower bound is met everywhere in the network. Note that these constraints also ensure that these power values are greater than $P_{1}$ if the link is not used by the light-forest. The significance of these conditions will be clear when they are used along with constraint (17) below.

$$
\begin{aligned}
& P_{i, j, d B m}^{b e g, a, \lambda} \leq P_{2} \times\left(1-T_{i, j}^{a, \lambda}\right) \\
& \forall e(i, j) \in E ; 0 \leq a<K ; \forall \lambda \in \Lambda
\end{aligned}
$$

Constraint (17) ensures that the power level at the beginning of each link is less than $P_{2}$ if the fiber is not part of a tree. The values of $P_{2}$ and $P_{1}$ are chosen to be much less than any power loss in the network, and $P_{2}>P_{1}$. Therefore, this constraint is used along with constraints (15) and (16) to guarantee that the power values are very small (between $P_{1}$ and $P_{2}$ ) everywhere on unused channels.

$$
\begin{aligned}
T P_{i, j, m W}^{b e g} \leq & T P_{M A X}^{m W} \times \Upsilon_{i, j}+T P_{M I N}^{m W} *\left(1-\Upsilon_{i, j}\right) \\
& \forall e(i, j) \in E ; 0 \leq a<K ; \forall e(i, j) \in E
\end{aligned}
$$

The above constraint ensures that the aggregate power level at the beginning of each fiber is below the $T P_{M A X}^{m W}$ if the link is used by any session; otherwise, it is $T P_{M I N}^{m W}$. Since $T P_{M I N}^{m W}$ is zero, we can remove the second term in the righthand side of the inequality. Also, there is no need to extend this constraint to every OA since enforcing this upper bound at the beginning of the link guarantees that the total input power at the first OA cannot exceed $T P_{M A X}^{m W}$. The OA gain model expressed by equation (1) guarantees that the output power from the OA does not exceed this upper bound.

Please note that the aggregate power values at the beginning of the links are expressed in $m W$ while the individual channel powers are expressed in $\mathrm{dBm}$. In order to convert between these two units, the following relation needs to be implemented in the formulation:

$$
\begin{aligned}
& P_{i, j, m W}^{b e g, a, \lambda, o}=10^{\frac{P_{i, j, d B m}^{b e g, a, \lambda, o}}{10}} \\
& \quad \forall e(i, j) \in E ; \forall \lambda \in \Lambda ; 0 \leq a<K ; \forall o \in O A_{i, j}
\end{aligned}
$$

Unfortunately, this relation is non-linear. Therefore, we introduce a special set of linear constraints that are equivalent to (19) and take the following format:

$$
\begin{aligned}
& P_{i, j, m W}^{b e g, a, \lambda, o} \geq A_{y} P_{i, j, d B m}^{b e g, a, \lambda, o}+B_{y} \\
& \quad \forall e(i, j) \in E ; \forall \lambda \in \Lambda ; 0 \leq a<K ; \forall y \in Y ; \forall o \in O A_{i, j}(20)
\end{aligned}
$$

The idea is to define a set, called $Y$, of piecewise linear segments such that each segment is represented by a linear equation, called $y$, whose value is greater than the nonlinear curve of the relation defined in (19) over a specific period denoted by $Z_{y}=\left[\alpha_{y}, \beta_{y}\right]$. $Z_{y}$ determines the domain over which $y$ is defined. In order to guarantee the correct representation of these segments, the following conditions should be satisfied:

1) $Z=\bigcup_{y \in Y} Z_{y}=\left[P_{S e n}^{d B m}, P_{M A X}^{d B m}\right]$

2) $\alpha_{y}=\beta_{y-1} \quad \forall y \in Y, \alpha_{y} \neq P_{S e n}^{d B m}$

3) $\beta_{y}=\alpha_{y+1} \quad \forall y \in Y, \beta_{y} \neq P_{M A X}^{d B m}$

The first condition states that the union of the domains of the individual segments should equal the total domain over which relation (19) is defined (namely, $\left[P_{S e n}^{d B m}, P_{M A X}^{d B m}\right]$ ). The second condition states that the first value in function $y$ 's domain equals the last value in the previous function's domain, except for the first segment since its value should equal $P_{S e n}^{d B m}$. Similarly, the third condition ensures that the last value in function $y$ 's domain equals the first value in the previous function's domain except for the last segment since its value should equal $P_{M A X}^{d B m}$.

As long as these conditions are satisfied, any set $Y$ can be defined over any set of sub-domains, and increasing the number of individual segment, i.e., $|Y|$, improved the accuracy of this approximation, but also increases the formulation complexity. To help the reader understand the idea behind this approximation, please refer to Figure 1 which shows an example such that $|Y|=3$ and $Z=$ $\{[-30,-20],[-20,-10],[-10,0]\}$. Assume that the power value that needs to be converted is $-5 \mathrm{dBm}$. The corresponding values computed from $y_{1}, y_{2}$ and $y_{3}$ are $0.0235 \mathrm{~mW}$, $0.145 \mathrm{~mW}$ and $0.55 \mathrm{~mW}$, respectively. Constraint (20) ensures that the corresponding $m W$ value is not less than the maximum value computed from the y's (i.e., the one that corresponds to $y_{3}$ in this case). With the assistance of the objective function defined in (2), the power value in $\mathrm{dBm}$ is successfully mapped to the exact value of the maximum power value. Please note that the convex nature of relation (19) ensures that exactly one function will have the maximum value within each domain. This function corresponds to the function used to represent this segment.

Also, it is worth mentioning that because of the convexity of equation (19), the power value in $m W$ computed by the constraints defined by (20) may exceed the correct value. For example, while $-5 \mathrm{dBm}$ corresponds to $0.55 \mathrm{~mW}$ using $y_{3}$, the exact value is $0.316 \mathrm{~mW}$ using the relation (19). However, slightly overestimating the power level is still acceptable and will result in correct operation since the purpose of this conversion is to only ensure that the total power on the fiber is not exceeded.

$$
\begin{gathered}
T P_{i, j, m W}^{b e g}=\sum_{\lambda \in \Lambda} \sum_{a=0}^{K-1} P_{i, j, m W}^{b e g, a, \lambda} \quad \forall e(i, j) \in E \\
T P_{i, j, m W}^{b e g, o}=\sum_{\lambda \in \Lambda} \sum_{a=0}^{K-1} P_{i, j, m W}^{b e g, a, \lambda, o} \\
\forall e(i, j) \in E ; \forall o \in O A_{i, j}
\end{gathered}
$$

Constraints (21) and (22) calculate the total power in $m W$ at the beginning of fibers and optical amplifiers, respectively, as the sum of the power values in $m W$ of the individual channels.

$$
\begin{aligned}
& P_{i, j, d B m}^{b e g, a, \lambda, 1}=P_{i, j, d B m}^{b e g, a, \lambda}-\beta \times L_{i, j}^{0,1} \\
& \quad \forall e(i, j) \in E ; \forall o \in O A_{i, j} ; 0 \leq a<K ; \forall \lambda \in \Lambda(23)
\end{aligned}
$$




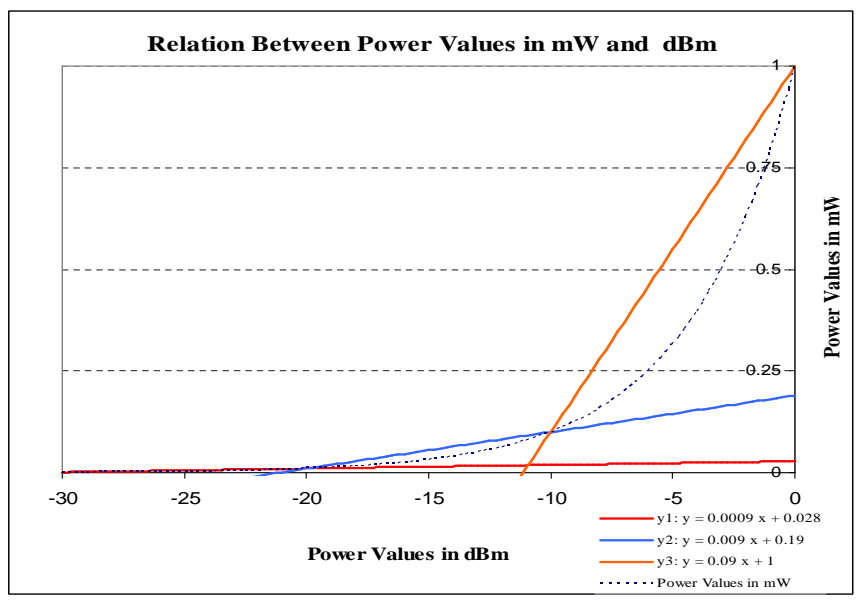

Fig. 1. An illustration of the approximate linear conversion approach between power levels in $\mathrm{dBm}$ and $m W$.

$$
\begin{gathered}
P_{i, j, d B m}^{e n d, a, \lambda}=\left[\left(1-N O A_{i, j}\right) \times\left(P_{i, j, d B m}^{b e g, a, \lambda}-\beta \times L_{i, j}\right)\right]+ \\
{\left[N O A_{i, j} \times\left(P_{i, j, d B m}^{b e g, a, \lambda,\left|O A_{i, j}\right|}+\right.\right.} \\
\left.\left.G_{i, j}^{o}-\beta \times L_{i, j}^{\left|O A_{i, j}\right|,\left|O A_{i, j}\right|+1}\right)\right] \\
\forall e(i, j) \in E ; \forall 0 \in O A_{i, j} ; 0 \leq a<K ; \forall \lambda \in \Lambda \\
P_{i, j, d B m}^{b e g, a, \lambda, o+1}=P_{i, j, d B m}^{b e g, a, \lambda, o}+G_{i, j}^{o}-\beta \times L_{i, j}^{o, o+1} \\
\forall e(i, j) \in E ; \forall O \in O A_{i, j} ; 0 \leq a<K ; \forall \lambda \in \Lambda
\end{gathered}
$$

The set of constraints (23 - 25) determines the power relation between the various optical components over each link. The constraints in (23) find the relation between the power at the beginning of the first $\mathrm{OA}$ and the beginning of the fiber link where the power decays due to propagation loss only. Equation (24) is used on link $e(i, j)$ to express the power strength on wavelength $\lambda$ at the end point of the link in terms of the power strength at the beginning of the link and the loss due to attenuation in the whole fiber length when $\left|O A_{i, j}\right|=0$. However, if $\left|O A_{i, j}\right|>0$, the power level at the end of the link is computed in terms of the power at the beginning of the last OA, its gain and loss due to attenuation in the last fiber segment. Finally, the set of constraints (25) finds the relation between the power at the beginning of an $\mathrm{OA}$ and the beginning of the previous one on the same fiber link in terms of its gain and the power attenuation in the fiber segment between two successive OAs.

$$
\begin{aligned}
& \left(1-T_{i, j}^{a, \lambda}\right) \times v+P_{i, j}^{e n d, a, \lambda}-S L_{j}^{a, \lambda}-\gamma \\
& \quad \geq\left(1-T_{j, k}^{a, \lambda}\right) \times w+P_{j, k}^{b e g, a, \lambda} \\
& \quad \forall e(i, j), e(j, k) \in E ; 0 \leq a<K ; \forall \lambda \in \Lambda \\
& \left(1-T_{i, j}^{a, \lambda}\right) \times w+P_{i, j}^{e n d, a, \lambda}-S L_{j}^{a, \lambda}-\gamma \\
& \quad \leq\left(1-T_{j, k}^{a, \lambda}\right) \times v+P_{j, k}^{b e g, a, \lambda} \\
& \quad \forall e(i, j), e(j, k) \in E ; 0 \leq a<K ; \forall \lambda \in \Lambda
\end{aligned}
$$

Constraints (26) and (27) relate the power values at the end of a fiber link, say $e(i, j)$ and the beginning of the following link, say $e(j, k)$, if any. In order to handle the various possibilities of usage of links $e(i, j)$ and $e(j, k)$, the values of

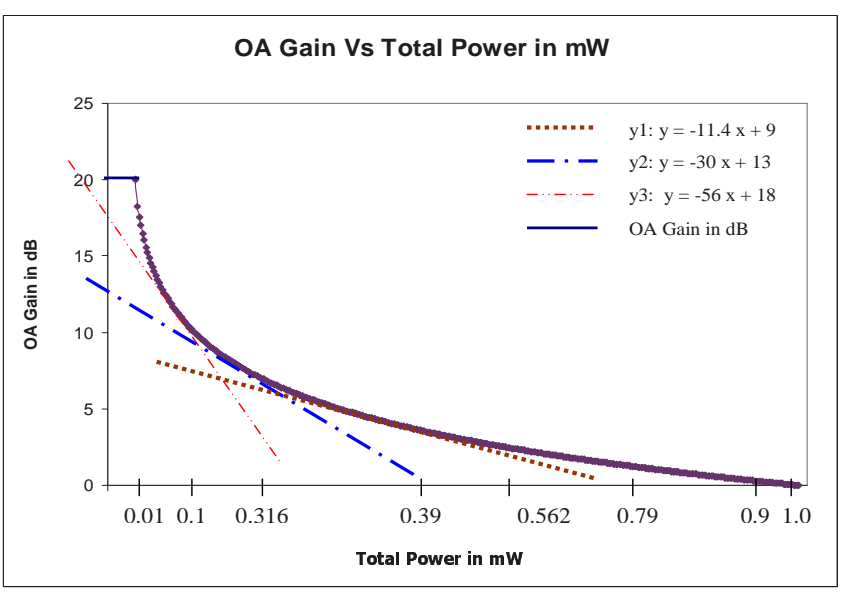

Fig. 2. An illustration of the approximate linear conversion approach between total input power of $\mathrm{OA}$ in $m W$ and the $\mathrm{OA}$ gain in $d B$

$v$ and $w$ are chosen such that $v \geq\left|P_{1}\right|+M$, and $w<0$. For example, consider the case when both $T_{i, j}^{a, \lambda}$ and $T_{j, k}^{a, \lambda}$ equal 0 . Constraints (15)-(17) set the value of $P_{i, j}^{b e g, a, \lambda}$ and $P_{j, k}^{b e g, a, \lambda}$ to be between $P_{1}$ and $P_{2}$. Choosing $v$ to be equal to $(6 \times M)$ guarantees that the left hand side of inequality (26) is always greater than the right hand side even when both power values are equal to $P_{1}$. The same holds for the left hand side of inequality (26). Choosing the value of $w$ to be negative helps in ensuring this too. This criterion for choosing $v$ and $w$ holds for all the other three cases of link usage.

We also use another mapping scheme to compute the OA gain (in $\mathrm{dB}$ ) from the total power (in $m W$ ) at the OAs' entry points. Using the OA model defined by Equation (1), the relation used in this mapping is shown in Figure 2. The set of linear equations used in this approximation are chosen such that the resulting value is less than the actual gain value (as depicted by the dashed lines in Figure 2). Because the small signal gain, $G_{0}$, is achieved only when the total input power does not exceed certain value (called $\left.P_{\text {sat }}\right)^{1}$, the mapping needs to handle two operating zones of the OAs. Therefore, we use the binary variable $A P_{i, j}^{o}$ to determine whether the $o^{t h}$ OA operates in the saturation region or not. $A P_{i, j}^{o}$ is ensured to be 1 if the total input power in $m W$ is less than $P_{\text {Sat }}$, and zero otherwise, using the following set of constraints:

$$
\begin{aligned}
\left(P_{S a t}-T P_{i, j, m W}^{b e g, o}\right)+\delta \leq A P_{i, j}^{o} & \\
\forall e(i, j) & \in E ; \forall o \in O A_{i, j}
\end{aligned}
$$

$A P_{i, j}^{o}$ is then used in constraints (29) and (30) to convert between $T P_{i, j, m W}^{b e g, o}$ and $G_{i, j}^{o}$.

$$
\begin{gathered}
\left(1-A P_{i, j}^{o}\right)+\frac{G_{i, j}^{o}-G_{0}}{M} \geq 0 \quad \forall e(i, j) \in E ; \forall o \in O A_{i, j} \\
A P_{i, j}^{o}+\frac{G_{i, j}^{o}-\left(A_{y} \times T P_{i, j, m W}^{b e g, o}+B_{y}\right)}{M} \geq 0 \\
\forall e(i, j) \in E ; \forall o \in O A_{i, j}
\end{gathered}
$$

${ }^{1}$ The values used in Figure 2 for $P_{S a t}$ and $G_{0}$ are $0.01 \mathrm{~mW}$ and $20 \mathrm{~dB}$, respectively. 
While constraint (29) considers the maximum gain region, constraint (30) handles the linear mapping for the saturation region in a fashion similar to the one proposed in (20).

$$
\frac{S L_{i}^{a, \lambda}}{M} \leq S P_{i} \quad \forall i \in N ; 0 \leq a<K ; \forall \lambda \in \Lambda
$$

Constraint (31) ensures that the splitting at an MI node is $0 d B$ for any connection carried on any channel. However, the splitting loss in $d B$ at MC nodes is determined by this relation:

$$
S L_{i}^{a, \lambda}=10 * \log _{10} f
$$

This relation is non-linear since the splitting degree, $f$, is a variable. Hence, we introduce the following set of linear equations that are equivalent to (32).

$$
\begin{aligned}
& \frac{\sum_{j, j \neq s r c_{a}, e(i, j) \in E} T_{i, j}^{a, \lambda}-f+\delta}{M} \leq A_{f} \\
& \quad \forall i \in N ; 0 \leq a<K ; \forall \lambda \in \Lambda ; 2 \leq f<\text { Out }_{i} \\
& A_{f} \times\left\{10 \times \log _{10} f\right\} \leq S L_{i}^{a, \lambda} \\
& \quad \forall i \in N ; 0 \leq a<K ; \forall \lambda \in \Lambda ; 2 \leq f<O u t_{i} \\
& S L_{i}^{a, \lambda} \geq 0 \quad \forall i \in N ; 0 \leq a<K ; \forall \lambda \in \Lambda
\end{aligned}
$$

The value of $A_{f}$ is 1 for all the values of $f$ that are less than or equal the actual tree fanout at the node; otherwise it can be either 0 or 1 . However, with the help of the objective function (2), which minimizes the individual amplifier gains, this helps to minimize the fanout, and hence the splitting loss, $S L_{i}^{a, \lambda}$. Therefore, the value of $A_{f}$ will be set to 0 when the value of $f$ is greater than the actual tree fanout.

\section{The Heuristic Algorithm}

The complexity of the MILP formulation presented in the previous section is on the order of $2 \times N^{2} \times K \times|\Lambda| \times|O A|$ for both the number of variables and constraints. Due to its high complexity, solving this MILP using the conventional solvers is not scalable for large network and/or traffic sizes. Using parallelized MILP solvers can help overcome this scalability issue. However, due to the high-cost associated with accessing and using these advanced solvers, there is a need for a heuristic algorithm that can provide faster, yet accurate solutions.

We present a heuristic approach for the PA-RWA problem under AOM service. We refer to this approach as Power-Aware Multicasting (PAM) algorithm. The heuristic decomposes the PA-RWA problem into three subproblems, i.e., Routing (R), Wavelength Assignment (WA) and Power Assignment (PA) subproblems. Although solved separately, the PAM heuristic correlates these subproblems by using a special set of cost functions for the links, network and sessions.

\section{A. Motivation of the PAM Algorithm and Main Characteristics}

Call blocking can be due to many reasons, including powerrelated issues. The lack of availability of a continuous wavelength on the light-trees is the main non-power related source of call blocking. We refer to this type of call blocking as Wavelength-Shortage Blocking. On the other hand, powerrelated reasons of call blocking include the case when the source nodes fail to launch their light-trees with the appropriate power levels without violating any of the power constraints described in Subsection III-A. This can even happen without lack of network resources and wavelengths. Hence, this type of blocking is called Power-Sharing Blocking and it takes three formats:

1) Power-Shortage Blocking, which occurs if the power strength of the signal drops below $P_{S e n}^{d B m}$ anywhere in the light-tree. This drop prohibits the detection of the optical signal at receivers and OAs.

2) Service-Disruption Blocking, which results when assigning a power value to a new session yields a sequence of changes in the strength of the already launched optical signals and OAs gains which results in violating the power constraints. In this case, the new session is blocked.

3) Non-linear Impact Blocking, which is a direct result of violating the maximum total power constraint. Allowing this to happen causes the system to operate in the undesirable nonlinear region of the fiber ${ }^{2}$.

The PAM algorithm is designed to capture the impact of the various Power-Sharing Blocking sources. This is achieved in part by using a novel cost function for the links, sessions and network. Also, the PAM algorithm has two characteristics. First, the algorithm deals with the sessions in a grouping fashion, rather than individually, by treating a subset of the sessions together during the same iteration. This grouping mechanism provides flexibility for the algorithm to deal with the interaction between the sessions which can result in reducing blocking. Second, the power module of the proposed algorithm adopts a semi-random scheme, that is controlled by a set of probabilities that are used to determine the best combination of power levels at various source nodes. This semi-random power assignment scheme accompanied with the grouping mechanism provide better solutions when compared to the minimum power assignment and sequential treatment approaches.

\section{B. Link Cost Function}

We first define the following set of parameters are defined first. $P_{i, j, m W}^{\lambda, M I N}$ is the minimum power (in $m W$ ) for channel $\lambda$ that has to be observed at the beginning of link $e(i, j)$ in order for this signal to reach the first ${ }^{3} \mathrm{OA}$ (if $\left|O A_{i, j}\right|>0$ ), or the end of the link (if $\left|O A_{i, j}\right|=0$ ) with a power that is exactly equal to $P_{S e n}^{m W}$.

$\left|\Lambda_{i, j}^{M A X}\right|$ determines the maximum number of wavelengths that link $e(i, j)$ can ever support. This upper bound is achieved when all the connections carried over the link are one-hop unicast connections from node $i$ to node $j .\left|\Lambda_{i, j}^{M A X}\right|$ is then calculated as:

$$
\left|\Lambda_{i, j}^{M A X}\right|=\operatorname{MIN}\left(|\Lambda|, \frac{T P_{M A X}^{m W}}{P_{i, j, m W}^{\lambda, M I N}}\right)
$$

\footnotetext{
${ }^{2}$ Although this blocking behavior can be prevented with the use of equalizers, like in [8][9], our system model does not assume the use of equalizers.

${ }^{3}$ For the sake of simplicity, the definition of $P_{i, j, m W}^{\lambda, M I N}$ deals with the first OA over one link only.
} 
Therefore, $\left|\Lambda_{i, j}^{M A X}\right|$ is determined, not only by the wavelengths availability, but also by the signals ability to reach the first OA, if any, and still be detectable.

Given the current power assignments at the beginning of link $e(i, j)$, we define $\left|\Lambda_{i, j}^{A d d}\right|$ as the number of wavelengths that can additionally be supported over the link. $\left|\Lambda_{i, j}^{A d d}\right|$ is computed as:

$$
\operatorname{MIN}\left(\left\lfloor\frac{T P_{M A X}^{m W}-\sum_{\lambda \in\left|\Lambda_{i, j}^{\text {Busy }}\right|} P_{i, j, m W}^{\text {beg,a, },}}{P_{i, j, m W}^{\lambda, M I N}}\right\rfloor,\left|\Lambda_{i, j}^{F r e e}\right|\right)
$$

where, $\left|\Lambda_{i, j}^{\text {Busy }}\right|$ and $\left|\Lambda_{i, j}^{\text {Free }}\right|$ represent the number of used and unused channels over $e(i, j)$, respectively. From equations (36) and (37), one should conclude that $\left|\Lambda_{i, j}^{A d d}\right| \leq\left|\Lambda_{i, j}^{M A X}\right| \leq|\Lambda|$.

The current cost of $e(i, j), c_{e(i, j)}$, is then defined as the ratio of the maximum number of wavelengths supported over $e(i, j)$ and the current potential available wavelengths, namely,

$$
c_{e(i, j)}=\frac{\left|\Lambda_{i, j}^{M A X}\right|}{\left|\Lambda_{i, j}^{A d d}\right|}
$$

Equation (38) has the following properties:

- It intelligently incorporates the useful concepts of power sharing and wavelength availability into the link cost, which is used in the $\mathbf{R}$ phase. This provides the necessary mechanism to take the impact of the WA and PA subproblems on the $\mathbf{R}$ subproblem into consideration.

- It is a dynamic function, i.e., link costs change with the accumulated power values. Hence, routing decisions are directly dependent on the changing network status.

- It is a positive increasing function, i.e., link costs increase with the increase in the link usage. This results in balancing the load in the entire network.

Also, we define the network cost as $\sum_{e(i, j) \in E} c_{e(i, j)}$. In order to take the impact of provisioning a light-forest on the remaining unprovisioned ones, the cost of each light-forest is calculated as the increase in the network cost. Finally, selecting the sessions to be provisioned in the network is governed by an ordering policy that sorts the sessions based on their cost. Choosing the ordering policy is an implementation preference; namely, it is not PAM algorithm specification.

\section{The PAM Algorithm Details}

The basic operation of the PAM algorithm is depicted in Figure 3. The output of the algorithm is the number of admitted sessions, and their corresponding RWA and power values (or RWA-P). The core of the PAM algorithm is the RWA-PAM Stage. The input to this stage is the set of sessions to be provisioned $(\mathrm{CC})$, the sharing degree and the construction mode. The sharing degree represents the number of sessions to be considered by the RWA-PAM stage. For example, if the sharing degree is 1, the RWA-PAM stage constructs the RWA$\mathrm{P}$ solution for one light forest at a time.

In order to calculate the cost parameters defined in Subsection V-B, PAM algorithm keeps track of the network status parameters, which consist of, the RWA for each provisioned connection, set of blocked connections, the wavelength availability and the power values over each channel at every

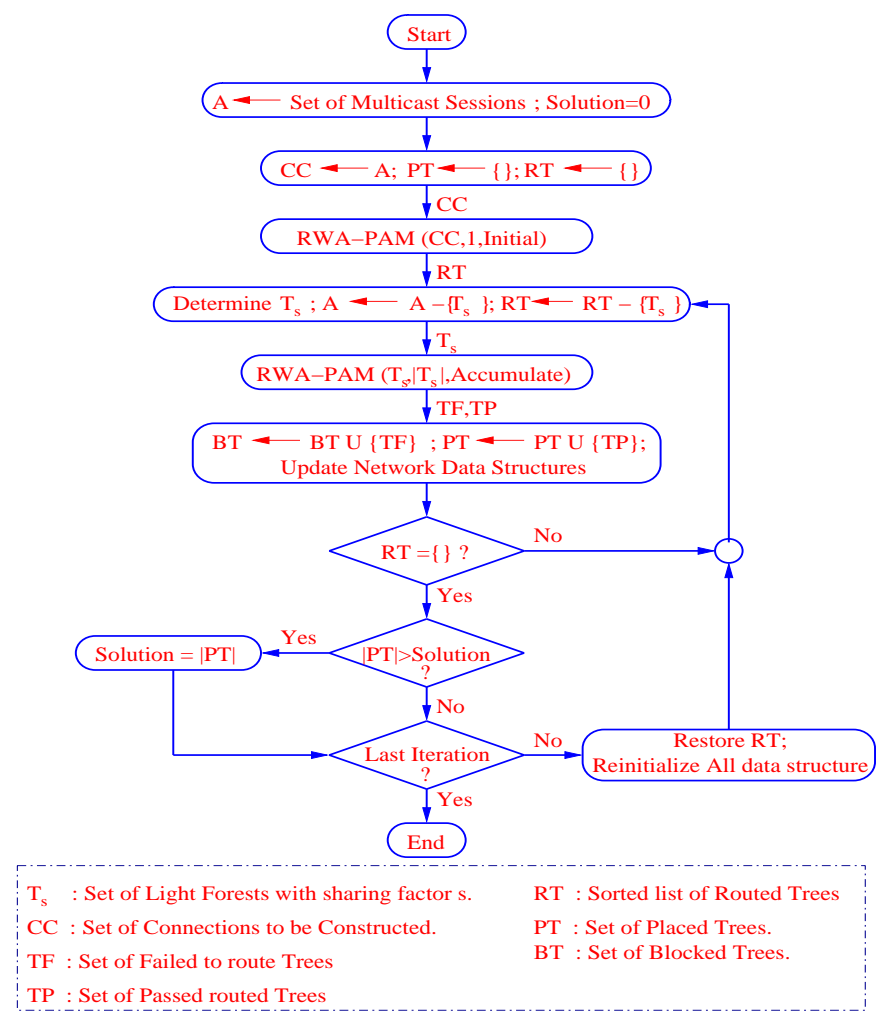

Fig. 3. Flow chart of the PAM Algorithm Operation.

network location. Therefore, after each iteration, the network status parameters are updated in order to reflect their usage by the provisioned sessions, if any. For example, if one session is provisioned at the iteration, it will be added to the list of provisioned sessions, and the set of available wavelengths is updated to exclude the wavelength(s) used over each link of its light-forest. Similarly, the power strength of each assigned channel is updated over each light-forest's link.

The RWA-PAM stage of the algorithm defines two construction modes based on the age $\mathrm{e}^{4}$ of the power status information with respect to each iteration. In the first mode, each session's RWA-P is constructed using the initial network status that existed before provisioning any session. Therefore, this mode is referred to as the Initial mode as the algorithm operates using a fresh network state with no used resources. The second mode, called the Accumulate mode, takes the cumulative impact of the provisioned sessions on the network power status by relying on the latest status information, rather than the initial counterpart.

Accordingly, the PAM algorithm operates in two phases. In the first phase, called the Initial Phase, RWA-PAM stage operates using the Initial mode in order to identify the set of sessions that are blocked due to the given OA placement in the network, and hence eliminate them from further investigation. Such physical limitation cannot be circumvented and it occurs with or without the existence of other sessions in the network.

The second phase of the PAM algorithm is called the

\footnotetext{
${ }^{4}$ The age of the power information denotes the iteration at which the power values in the network are considered as a reference when computing the RWA$\mathrm{P}$ solutions at each iteration. The RWA-P solutions will be more accurate if they are based on the latest (fresh) power values in the network.
} 


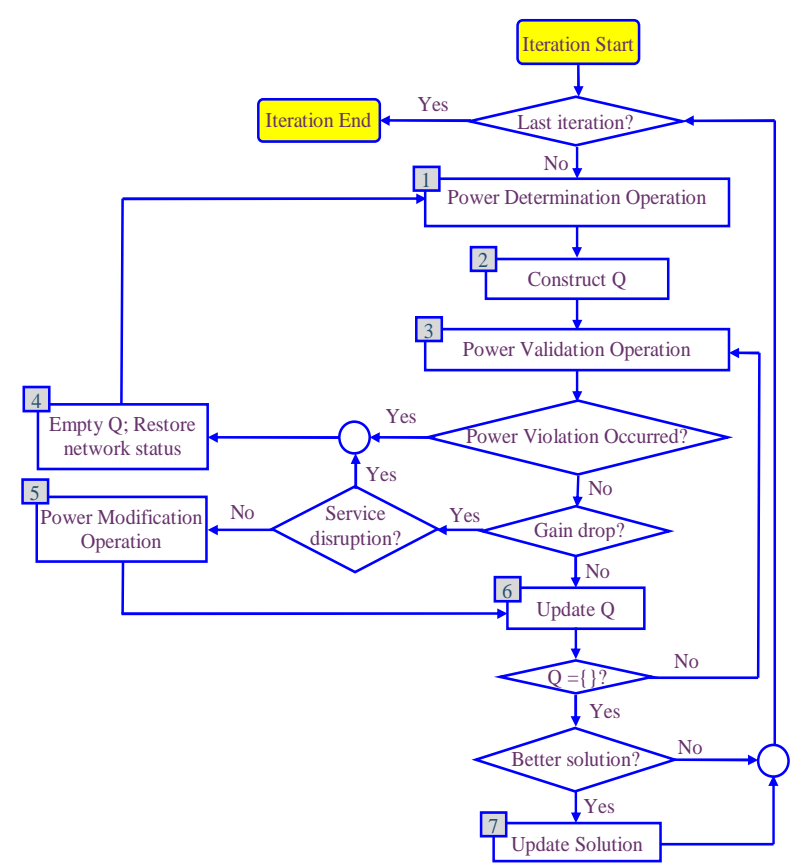

Fig. 4. Flow chart of the operation of Power Assignment (PA) Module. The number associated with each task box is used in the algorithm description in Subsection V-D to refer to the corresponding operation.

Iterative Phase. During each iteration of this phase, the RWAPAM stage operates in the Accumulate mode and the number of sessions considered depends on the sharing degree. The solution found from each iteration is computed with respect to the recent network status which is re-initialized before the next iteration starts. Finally, the best solution out of all the iterations is selected to be the final solution.

The RWA-PAM stage performs three main operations, namely, routing, wavelength assignment and power assignment. The algorithm supports two routing operation modes, i.e., Fixed and Adaptive. With the Fixed mode, the routing operation is performed in the Initial Phase only; therefore, no modification in the light-forest structure is allowed thereafter. However, the Adaptive mode allows the routing operation to occur in the Accumulate Stage too in order to recompute the light-forest for the set of sessions that are not yet provisioned.

Routing is performed using an extended version of the Member-Only Heuristic (MOH)[18]. In $\mathrm{MOH}$, the multicast tree is expanded iteratively by including at least one member node per iteration. Tree expansion is done via a node set, which includes the source node, MC nodes and MI leaf nodes. This set is updated at each iteration. Instead of using a single path, we use $k$-shortest paths, where $k$ is an input parameter, such that the member node is connected to the tree through the path that causes the least increase in the network cost and which has sufficient power to reach the destination.

The Wavelength Assignment operation is performed using the First-Fit scheme in which the first available wavelength over the tree links is chosen. The details of the Power Assignment module is more involved and it is described in the next subsection, and its operation is shown in Figure 4.

\section{Power Assignment (PA) Module}

Designing the Power Assignment (PA) module relies on two important elements. First, it separates the links identity from their power values by using a queue structure, called $Q$, which consists of unique (un-repeated) instances of the links. Second, we adopt a semi-random iterative scheme for determining the power values at the source nodes. This scheme is based on a set of probabilities that determine both the update action and the amount of power change.

The PA module runs for a number of iterations. Given the power values of the already provisioned sessions, each iteration determines the best power values to be launched at the source nodes of the sessions under investigation. Each iteration consists of the following main steps (or operations):

1) Power Determination, which determines the set of power values (called Power Vector) at the source nodes.

2) Power Validation, which determines if this Power Vector violates any power constraints or results in changing the OAs gain over the links.

3) Power Modification, which is needed to determine if a gain drop can be tolerated in the network and no previously provisioned session is disrupted.

These operations are depicted in Figure 4 by boxes 1, 3 and 5 , respectively. For ease of reference in describing the module here, we will use these numbers, as well as the others in Figure 4, when describing the tasks performed in each step.

In the first iteration, the Power Vector is initialized in step 1 with the least operational power values at the source nodes of the sessions under investigation ${ }^{5}$. In step $2, Q$ is constructed by populating it with all the outgoing links from these source nodes. In step 3, the module proceeds on a link by link basis, starting from the link at the queue head and it checks if power levels are valid at all points on this link. If so, step 6 is invoked, in which the link is removed from $Q$ while its outgoing links are added to $Q$ if they are part of the light-tree of the sessions under consideration. At each algorithm step, $Q$ contains one instance of the link, even if it is shared by more than one light-tree. PA module continues with the next link in $Q$, and steps 3 and 6 are repeated. It stops when $Q$ becomes empty.

Please note that if a link is shared by multiple sessions, it can be revisited more than once during the iteration because it can be at different depths in the various light-trees. Therefore, not all the (final) values of the power strengths over this link are always available at the time PA module handles the link. The algorithm deals with those power values that are available. However, allowing several traversals of the links ensures the complete availability of the power values at the link.

Step 4 is invoked if the Power Validation operation in step 3 detects that either:

1) A violation of a power constraint is encountered at any point on the link, or

${ }^{5}$ The least operational power value is defined as the minimum power level that is required at the source node $(s r c)$ in order to reach the first OA, if any, with the least detectable power value, $P_{S e n}^{d B m}$. Without loss of generality, and assuming that the first tree link $e(s r c, j)$ has at least one OA, the least operational power value (in $d B m$ ) is computed as: $P_{S e n}^{d B m}+\beta * L_{s r c, j}^{s r c, 1}$, where $L_{s r c, j}^{s r c, 1}$ is the distance between $\operatorname{src}$ and the first OA on link $e(s r c, j)$. 
2) The gain of at least one OA drops to a level that disrupts the service of an already provisioned connection ${ }^{6}$.

In step 4, $Q$ is emptied, the network status is restored, and then the Power Determination operation in step 1 is invoked to determine the new Power Vector. The Power Determination operation becomes more involved now and the following tasks are performed:

1) The power violation type is determined as well as the affected sessions. The affected sessions here are determined by all the sessions (either, provisioned or not at the current algorithm iteration) which are using the link where the power violation or service disruption took place.

2) A set of sessions are then randomly selected and are blocked from participating in the next cycle of the PA module.

3) For each participating session, the module determines whether it can reuse its original power value or not.

4) If the power value is to be changed, the algorithm then determines, randomly, whether to increase or decrease the current power based on the power violation type. The amount of power change is determined randomly too.

The randomness involved in this step is governed by fixed probabilities that are carefully chosen to reflect the method used to resolve each power violation type. For example, if the individual power (in $d B m$ ) drops below $P_{S e n}^{d B m}$, then it is more probable that increasing the power value at the source nodes can resolve this issue. Hence, increasing the power value is given high probability in this case. On the other hand, service disruption is not always due to high power values. For example, decreasing the power at the source node might increase the gain of one OA, which increases its output power. This results in more input power in the following OA, which might decrease its gain and causes service disruption to persist. Therefore, we assign a moderate value to the probability of increasing the power value in the case of Service disruption. Finally, this probability is low in the case when total power (in $m W$ ) exceeds $T P_{M a x}^{m W}$.

In the case in which the drop in the OA gain does not cause service disruption, the Power Modification operation (step 5) is then invoked. In this case, the PA module first identifies the affected sessions (either provisioned or not) that currently use the link under investigation. It then proceeds to step 6 by populating $Q$ with links from the affected sessions set.

Due to the random nature of this solution, the PA module runs for a number of iterations (or if the solution does not improve within some limits). The maximum number of admitted sessions over all of these steps is chosen as the final PA module solution. Therefore, after each iteration, the solution is updated in step 7 if more sessions are admitted.

\section{Numerical Results}

We present some numerical results in this section. We solved the MILP formulation using CPLEX linear solver package

\footnotetext{
${ }^{6}$ Even though service disruption is the result of the power constraints violation, we treat it here separately as it has special treatment in determining the power vector in step 1, as will be explained later.
}

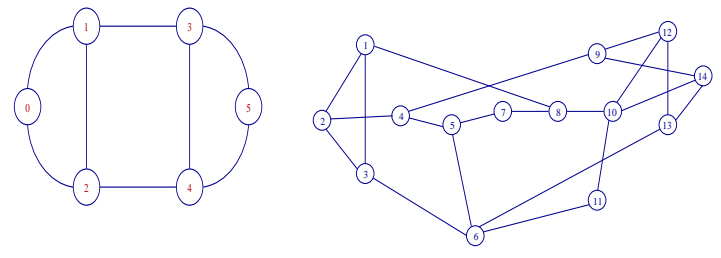

Fig. 5. Six Nodes Fig. 6. NSFNET. Network.

[19]. CPLEX experiments were conducted using the sample 6node mesh network shown in Figure 5. On the other hand, the PAM algorithm is implemented using the $\mathrm{C}++$ Programming Language and the simulation results were collected over the 14-nodes NSFNET shown in Figure 6. Table I demonstrates the numerical values for the basic system parameters used in our experiments. Also, the following assumptions were used for setting up the experiments:

1) The group size of each multicast session follows a uniform distribution between 1 and $N$, where $N$ is the number of nodes in the network.

2) Node membership in each multicast session group is determined using a uniform distribution over all the nodes except the session's source node.

3) The increasing order policy is used to determine the set of sessions to be provisioned based on their costs.

4) Each problem instance is represented by a set of sessions, network topology, and wavelength channels. Changing any of these parameters represents a new problem instance.

5) For each problem instance defined above, we run 6 independent experiments of the PAM algorithm. These experiments are conducted on the same problem instance, and the experiment that results in the maximum number of accepted sessions is then chosen as the representative solution for the problem instance.

6) The probability value that is used to increase the strength of the individual sources when the power value drops below $P_{S e n}^{d B m}$ is 0.9 . Similarly, this probability is chosen to be 0.5 or 0.1 when service disruption or exceeding maximum power level occurs, respectively.

The results in this section are presented in two parts. The first part establishes the quality of the solutions produced by the PAM algorithm. This is done by comparing these results by their optimal counterparts while using the 6-node network of Figure 5. In the second part, we quantify some basic characteristics of the PAM algorithm using the simulations results on the NSFNET in Figure 6.

\section{A. Solution quality of the PAM algorithm}

Figure 7 depicts the relative performance of the PAM algorithm compared to the optimal solutions from CPLEX when the number of available channels is 4 . Similar behavior is observed with other numbers of channels too. This relative performance is measured as the ratio between the number of sessions accommodated by the PAM heuristic and by the MILP formulation for a given number of sessions, denoted as, $K$. We 
TABLE I

TyPical VAlues for The System Parameters. $M$ CAN TAKe ANy VALUE THAT SATISFIES THE FOLLOWING CONDITION: $M>\operatorname{MAX}\left\{N^{2} *|\Lambda|, G_{0}\right\}$

\begin{tabular}{|c|c|c|c|c|c|}
\hline Symbol & $\beta$ & $P_{S e n}^{d B m}$ & $T P_{M A X}^{m W}$ & $\gamma$ & $G_{0}$ \\
\hline Value & $0.2 d B / k m$ & $-30 d B m$ & $1 m W$ & $1 d B$ & $20 d B$ \\
\hline \hline Symbol & $P_{1}$ & $P_{2}$ & $\delta$ & $v$ & $w$ \\
\hline Value & $-5 * M$ & $-2 * M$ & 0.01 & $20 * M$ & $-M$ \\
\hline
\end{tabular}

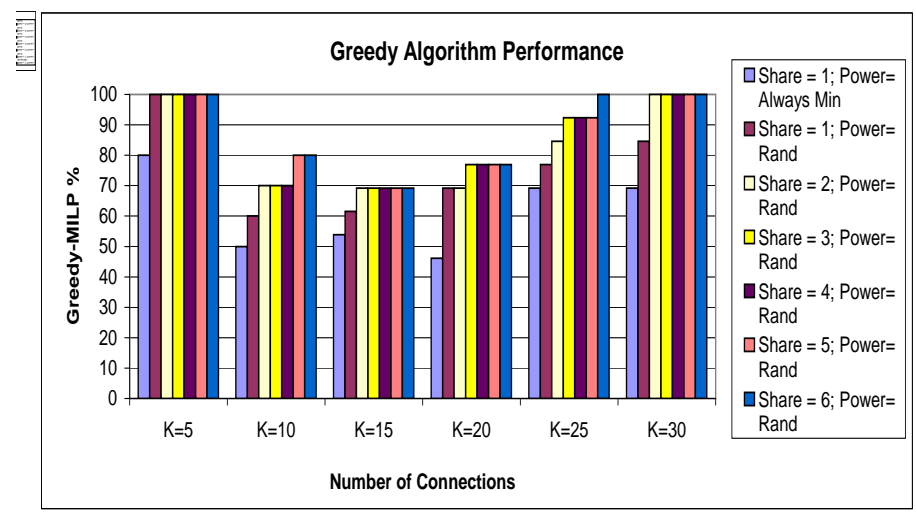

Fig. 7. The greedy heuristic results with respect to the MILP for the 6 nodes mesh network, when $\Lambda=4$ and for different values of sessions, $K$.

refer to this ratio here as the Greedy-MILP ratio. These results are obtained using the Fixed scheme with one alternate path. However, we allow different degrees of grouping (sharing) of sessions and we use different power assignment schemes.

When Sharing (Grouping) Degree, or simply Share, is 1, one session is provisioned at a time; otherwise, more than one session are considered. On the other hand, we refer to the semi-random power assignment method proposed in Section $\mathrm{V}$ as the Rand method. In Figure 7, we compare the Rand method with another deterministic scheme, referred to as the Min method. The Min method operates by always choosing the minimum possible power value.

As shown in Figure 7, the Greedy-MILP ratio is represented by a set of bars at each traffic load, $K$. Each bar represents the experiment settings of the simulation at that traffic load. The results represented by the first left bar for each $K$ (i.e., when $S=1$ and Min Power Assignment scheme is used) serves as a benchmark to measure the usefulness of the Share and Rand aspects of the PAM algorithm. The following conclusions can be drawn from this figure:

1) The PAM algorithm performs very well with respect to the corresponding optimal solutions. It provides near optimal solutions and sometimes was able to get the optimal solution. For example, the PAM algorithm produces the optimal solution (the Greedy-MILP ratio equals $100 \%$ ) when $K=30$ and Share $=2$ to 6 .

2) Applying the Rand power scheme results in a huge performance improvement compared to the Min Method. This improvement is measured as the increase in the value of the Greedy-MILP ratio. The maximum performance improvement occurs when the value of Share

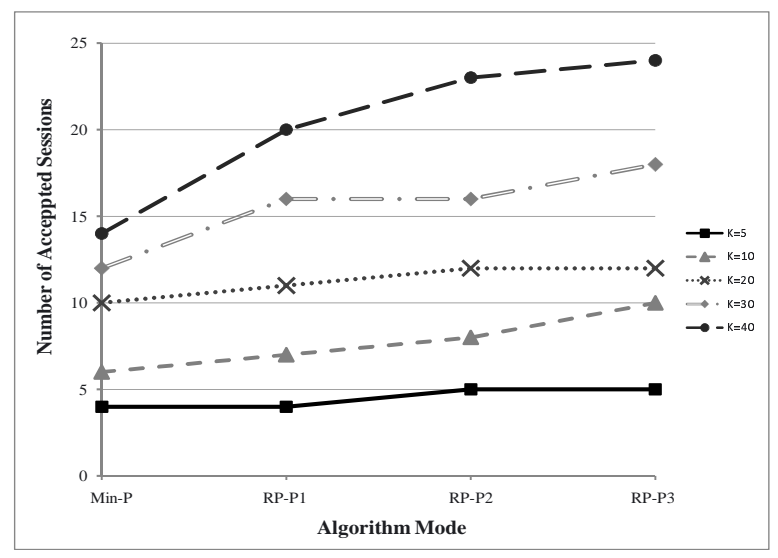

Fig. 8. Number of accepted sessions when Fixed Scheme is used and $\Lambda$ is 10. Min-P refers to case of Min Power scheme with one alternate path, while RP-P(i) refers to the case of Rand Power Scheme with $i^{\text {th }}$ alternate paths.

equals 1 and it ranges from $8 \%$ for $K=15$ and $K=25$, and $23 \%$ for $K=20$. However, this incremental improvement decreases as the sharing degree increases. This indicates that small sharing degrees are sufficient to reach an acceptable system performance that is close to the optimal solution. This has the advantage of reducing the system complexity since a fewer number of sessions per group can be considered at each algorithm iteration.

\section{B. PAM Heuristic Results}

This subsection is devoted to address the following characteristics of the PAM algorithm:

\section{PAM Algorithm's Impact on the Objective Function}

The impact of the PAM algorithm on the number of accepted sessions is depicted in Figure 8 at different traffic loads using the Fixed scheme and when the number of available channels is 10. Similar behavior is observed with the Adaptive scheme too. The results are collected at different computation demand. Each demand is determined by the power assignment scheme and the number of alternate paths. The least computation demand is the Min-P where the Min power assignment scheme is used with a single alternate path. The maximum computation demand is referred to as RP-P3 where Rand power assignment with 3 alternate paths routing is performed.

Naturally, the number of accepted sessions increases as the traffic load increases. However, it is obvious from this figure that for each traffic load, the objective function improves (i.e., increases) with the computation. For example, for $K=40$, the number of accepted sessions increases gradually from 14 to 24 when the algorithm mode increases from Min-P to RP-P3.

\section{Relative Performance of the Fixed and Adaptive Schemes}

We compare the performance of the Fixed Scheme to that of the Rerouting (Adaptive) ${ }^{7}$ scheme here. The comparison

\footnotetext{
${ }^{7}$ We use the term Rerouting to refer to the Adaptive scheme in this section as it emphasizes the core operation of this method.
} 
takes into account their performance in terms of the objective function and as well as the network resources usage. Two network resources are of interest for this comparison, namely:

1) $\kappa$, which determines the maximum number of distinct channels used over any link.

2) $€$, which determines the total number of links with at least one used channel.

The performance of each scheme in terms of the number of accepted sessions and network resources are shown in Tables II and III, respectively. From Table II, we find that the rerouting scheme always accepts higher number of sessions than that of the Fixed scheme at each alternate path. For instance, when $K=20$ and $\mathrm{P} 1$, the solution improves from 11 sessions to 13 sessions by using rerouting scheme.

Also, the results in Table II indicates that alternate routing and rerouting schemes work together in a constructive manner to find better solutions. For example, for the case when $K=20$, the solution improves from 11 sessions (when Fixed scheme is used with one alternate path) to 14 sessions (when Adaptive scheme is employed with 3 alternate paths).

However, as shown in Table III, the superior performance of the Adaptive scheme comes with a very minimal cost of consuming more network resources. This is because the Adaptive scheme responses dynamically to the changes in the network status and attempts to explorer bigger solution space which may require using more network resources. This behavior is different from the one reported in [8] and [9] where the Adaptive approach had better utilization for the network resources. This is directly due to the constrained wavelength availability nature of the problem we tackle here.

\section{Power Constraints' Impact on the Network Utilization}

Finally, we use PAM algorithm to investigate the impact of the power constraints on the network resources utilization.

TABLE II

NUMBER OF ACCEPTED SESSIONS FOR THE FIXED AND REROUTING SCHEMES WHEN $K=10,20$ AND 30.

\begin{tabular}{|c||c|c|c||c|c|c|}
\hline \multicolumn{1}{|c||}{} & \multicolumn{2}{c||}{ Fixed Scheme } & \multicolumn{3}{c|}{ Rerouting Scheme } \\
\hline \hline$K$ & 10 & 20 & 30 & 10 & 20 & 30 \\
\hline$P 1$ & 7 & 11 & 12 & 9 & 13 & 16 \\
\hline$P 2$ & 8 & 12 & 16 & 9 & 14 & 16 \\
\hline$P 2$ & 10 & 12 & 16 & 10 & 14 & 17 \\
\hline
\end{tabular}

TABLE III

CONSUMED NETWORK RESOURCES FOR FIXED (F) AND REROUTING (R) SCHEMES WHEN $K=10,20$ AND 30, AND 1 ALTERNATE PATH.

\begin{tabular}{|c|c|c|c|}
\hline$K$ & Mode & Max number of channels & Number of consumed links \\
\hline \hline 10 & $F$ & 3 & 21 \\
\hline 10 & $R$ & 4 & 23 \\
\hline \hline 20 & $F$ & 4 & 26 \\
\hline 20 & $R$ & 4 & 28 \\
\hline \hline 30 & $F$ & 4 & 32 \\
\hline 30 & $R$ & 6 & 35 \\
\hline
\end{tabular}

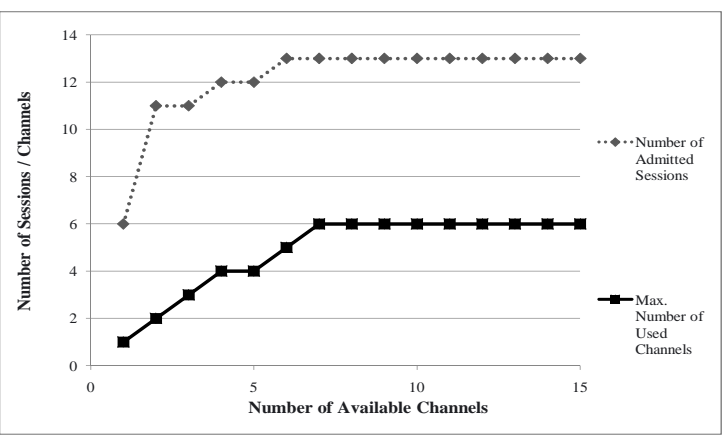

Fig. 9. System behavior with the existence of power constraints in the 6 nodes network for 30 connections.

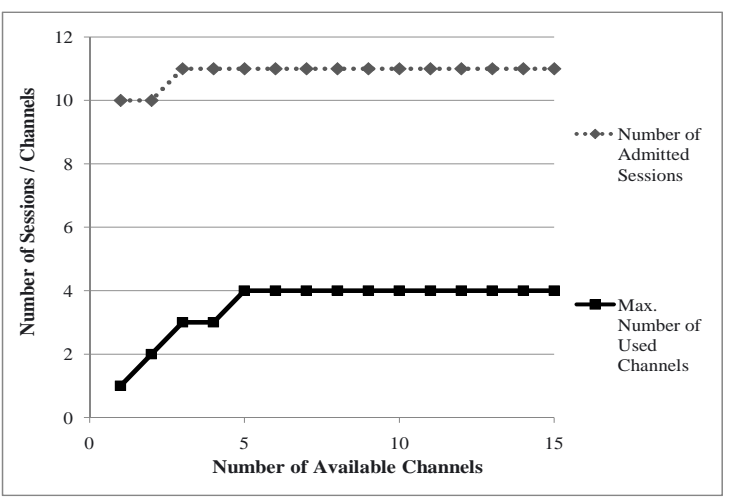

Fig. 10. System behavior with the existence of power constraints in the NSFNET for 20 connections.

The system throughput is determined in terms of the number of wasted channels due to the power constraints violation. Figures 9 and 10 depict the relation between the maximum number of admitted sessions and both the utilized wavelengths and the number of available wavelengths in the 6 node mesh network and the NSFNET, respectively. These experiments are conducted using the Fixed scheme with one alternate route with the total traffic load of 30 connections for the 6 mesh network and 20 connections for the NSFNET.

As shown in these figures, when the number of available channels is small, many sessions are dropped due to the wavelength shortage. The impact of the wavelength shortage continues until the number of available channels is less than 7 in the case of the 6 nodes network, and less than 5 in the NSFNET case. Before reaching this limit, increasing the number of available channels may reduce the system blocking probability if the main source of call blocking is the Wavelength-Shortage Blocking. For example, the number of admitted sessions increases from 6 to 13 as shown in Figure 9. However, this increase in the system performance stops when the Power-Sharing Blocking becomes the main source of call blocking and the OA placement [7] becomes the bottleneck for the PAM algorithm. At this point of time, increasing the number of channels does not improve the system blocking and these channels are wasted. Determining this cutoff value is 
essential for the network operators to determine the best usage of their network resources and the PAM algorithm provides a mechanism to provide such an important system metric.

\section{CONCLUSIONS}

We studied the problem of Power Aware Routing and Wavelength Assignment (PA-RWA) under All-Optical Multicasting (AOM) and we proposed two solution schemes. The first scheme produces optimal solutions using MILP formulation, while the other scheme proposed a heuristic scheme, called Power Aware Multicasting (PAM) algorithm. The design of the PAM heuristic consists of several optimization elements that enables it to produce near optimal solutions in an efficient manner. The results show that the PAM algorithm performs well in comparison with the optimal solutions. It also provides a good understanding and the network system. One of the main advantages of the proposed PAM Algorithm is its ability to determine the operation region for the network where adding more channels can benefits the overall system performance which is of great advantage for the network operators.

\section{ACKNOWLEDGMENT}

This research was supported in part by the National Science Foundation under grants ANI-0087746 and CNS-0626741.

\section{REFERENCES}

[1] H. Zang, J. Jue, and B. Mukherjee, "A review of routing and wavelength assignment approaches for wavelength-routed optical wdm networks," SPIE Optical Networks Magazine, vol. 1, no. 1, 2000.

[2] A. Hamad, T. Wu, A. Kamal, and A. Somani, "Multicasting protocols for wavelength routing networks," Computer Networks, vol. 50, no. 16, pp. 3105-3164, 2006.

[3] G. Rouskas, "Optical layer multicast: rationale, building blocks, and challenges," IEEE Netw., vol. 17, no. 1, pp. 60-65, 2003.

[4] L. Sahasrabuddhe and B. Mukherjee, "Light trees: optical multicasting for improved performance in wavelength routed networks," IEEE Commun. Mag., vol. 37, no. 2, pp. 67-73, 1999.

[5] D. Yang and W. Liao, "Design of light-tree based logical topologies for multicast streams in wavelength routed optical networks," in IEEE INFOCOM 03, vol. 1, pp. 32-41, 2003.

[6] M. Ali, B. Ramamurthy, and J. Deogun, "Routing and wavelength assignment with power considerations in optical networks," Computer Networks and ISDN Systems, vol. 32, no. 5, pp. 539-555, 2000.

[7] A. Hamad and A. Kamal, "Optimal power-aware design of all-optical multicasting in wavelength routed networks," in Proc. IEEE ICC 04, vol. 3, pp. 1796-1800, 2004.

[8] A. Hamad and A. Kamal, "Optical amplifier placement in wdm mesh networks for optical multicasting service support," IEEE/OSA Journal of Optical Communications and Networking, vol. 1, pp. 85-102, June 2009.

[9] A. Hamad and A. Kamal, "Efficient power-aware network provisioning for all-optical multicasting in wdm mesh networks," in IEEE GLOBECOM 08, pp. 1-5, 2008.

[10] B. Ramamurthy, J. Iness, and B. Mukherjee, "Optimizing amplifier placements in a multiwavelength optical lan/man: the unequally powered wavelengths case," IEEE/ACM Trans. Netw., vol. 6, no. 6, pp. 755-767, 1998.

[11] B. Ramamurthy, J. Iness, and B. Mukherjee, "Optimizing amplifier placements in a multiwavelength optical lan/man: the equally poweredwavelengths case," J. Lightw. Technol., vol. 16, no. 9, pp. 1560-1569, 1998.

[12] A. Fumagalli, G. Balestra, L. Valcarenghi, M. John, and C. Qiao, "Optimal amplifier placement in multi-wavelength optical networks based on simulated annealing," in Opt. Engr., vol. 3531, pp. 268-279, 1998.
[13] A. Sripetch and P. Saengudomlert, "Optimization for optical network designs based on existing power grids," IEICE Transactions on Communications, vol. E91-B, no. 3, pp. 689-699, 2008.

[14] J. W. K. Wu and C. Yang, "Multicast routing with power consideration in sparse splitting wdm networks," in IEEE Inter. Conf. on Communi. (ICC01), vol. 2, pp. 513-517, 2001.

[15] X. Yufeng and G. Rouskas, "Multicast routing under optical layer constraints," in IEEE INFOCOM 04, 2004.

[16] G. Markidis, S. Sygletos, A. Tzanakaki, and I. Tomkos, "Impairment aware based routing and wavelength assignment in transparent long haul networks," Lecture Notes in Computer Science, vol. 4534, pp. 48-57, 2007.

[17] R. Ramaswami and K. N. Sivarajan in Optical Networks: A Practical Perspective, Morgan Kaufmann, 2002.

[18] X. Zhang, J. Y. Wei, and C. Qiao, "Constrained multicast routing in wdm networks with sparse light splitting," J. Lightw. Technol., vol. 18, no. 12, pp. 1917-1927, 2000.

[19] http://www.ilog.com/products/cplex/. 